\title{
Recurrent Metastatic Melanoma Despite Multiple Surgical Interventions and Biological Targeted Therapy
}

\section{Hanieh Asadi, Metin Nizamoglu and Naguilb El-Muttardi}

St Andrew's centre for Plastic surgery, Chelmsford, United Kingdom

Corresponding author: Metin Nizamoglu, St Andrew's centre for Plastic surgery, Chelmsford, United Kingdom, Tel: 07702225711; E-mail: mnizam@doctors.org.uk

Received: 26 July 2016; Accepted: 28 July 2016; Published: 30 July 2016

Citation: Asadi H, Nizamoglu M, El-Muttardi N. Recurrent Metastatic Melanoma Despite Multiple Surgical Interventions and Biological Targeted Therapy. Arch Can Res. 2016, 4: 3

\section{Case Blog}

A 40 year old Caucasian male was referred to our plastic surgery unit with a rapidly growing mass in his left groin (Figure 1)

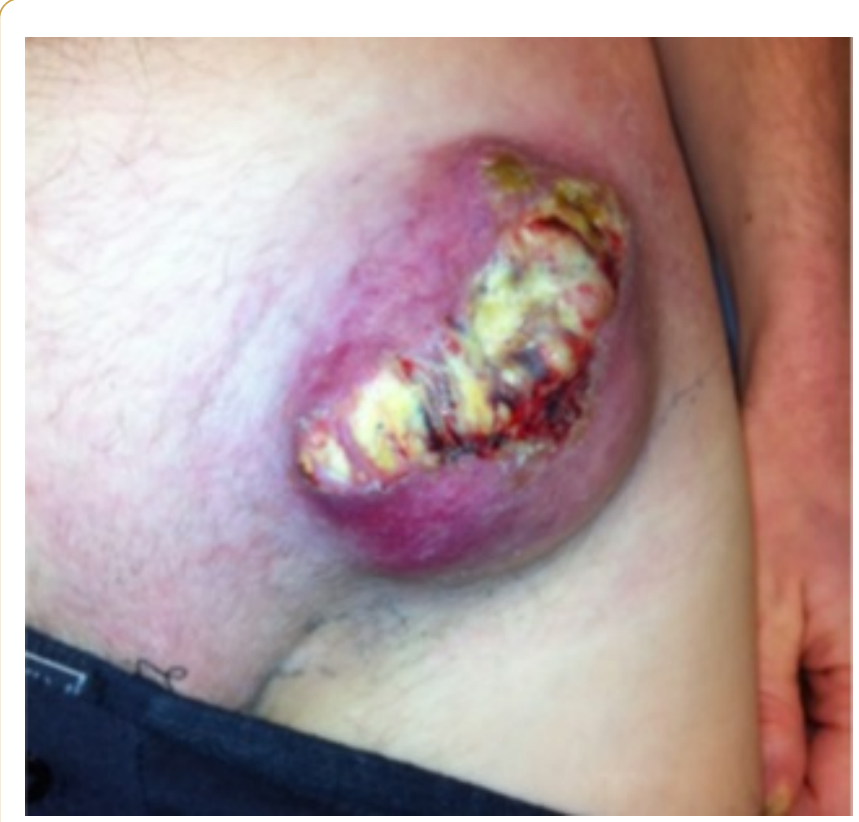

Figure1 Rapidly growing mass in his left groin.

A biopsy was taken which showed the lesion was metastatic amelanotic melanoma. No primary was identified. He had a history of a halo naevus on his lower back that had fully regressed (Figure 2).

A CT scan showed no systemic disease. The lesion was excised and reconstructed with a Tensor Fascia Lata flap (Figures $\mathbf{3}$ and 4). He also underwent ilio-inguinal lymph node dissection. The histology confirmed metastatic melanoma with clear excision from the peripheral margins and $2 \mathrm{~mm}$ from the deep margin.

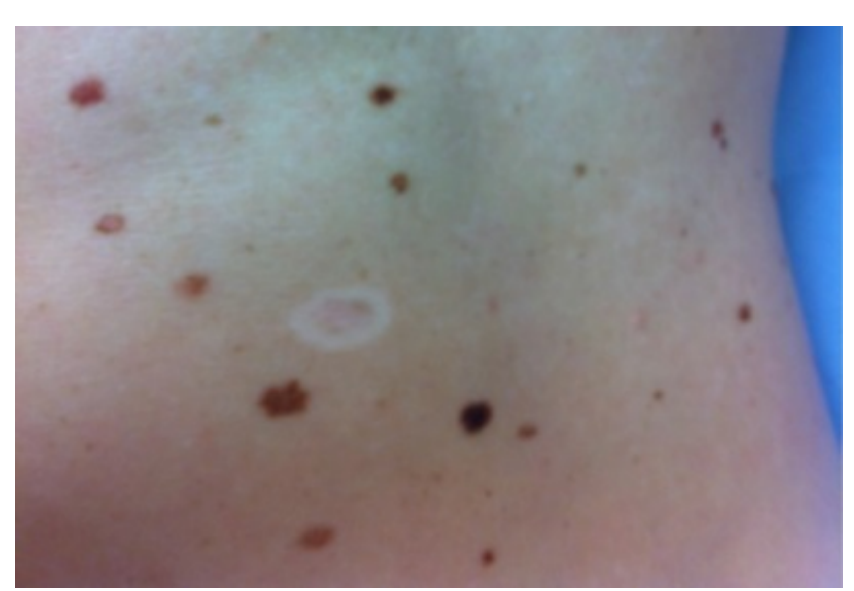

Figure $\mathbf{2}$ halo naevus on his lower back that had fully regressed.

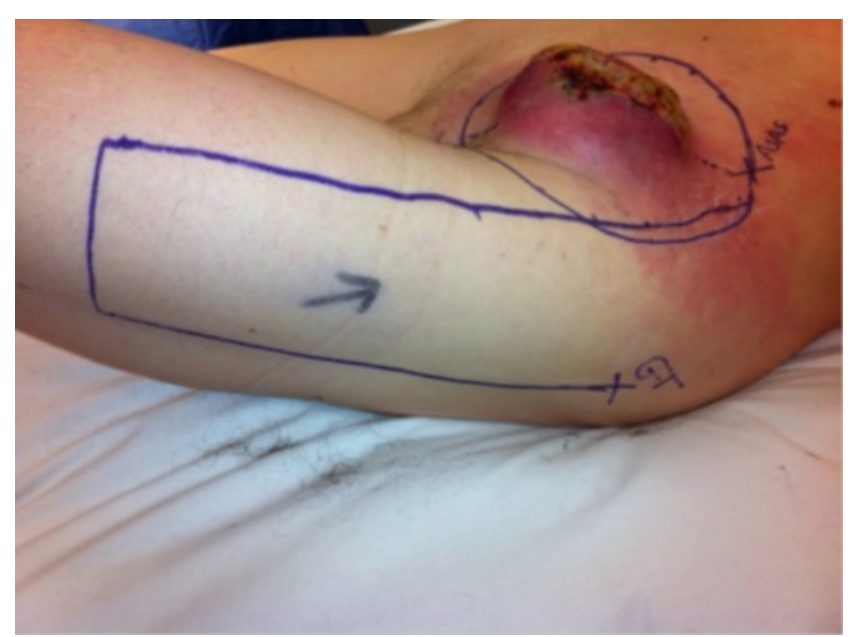

Figure 3 One month postoperatively a CT scan proved recurrence in the abdominal wall, suprapubic region, buttock, left inguinal region and right groin.

The patient underwent a right groin lymph node dissection and excision of subcutaneous metastases. The right groin dissection revealed $1 / 14$ lymph nodes positive for metastatic 
disease. The specimen was BRAF V600 positive. Treatment with Vemurafenib was commenced, within 2 weeks the cutaneous disease and nodal metastases started to regress with a reported significant improvement in overall health. However he developed erythroderma as a reaction to the Vemurafenib, which was halted then restarted at a lower dose once the reaction resolved.

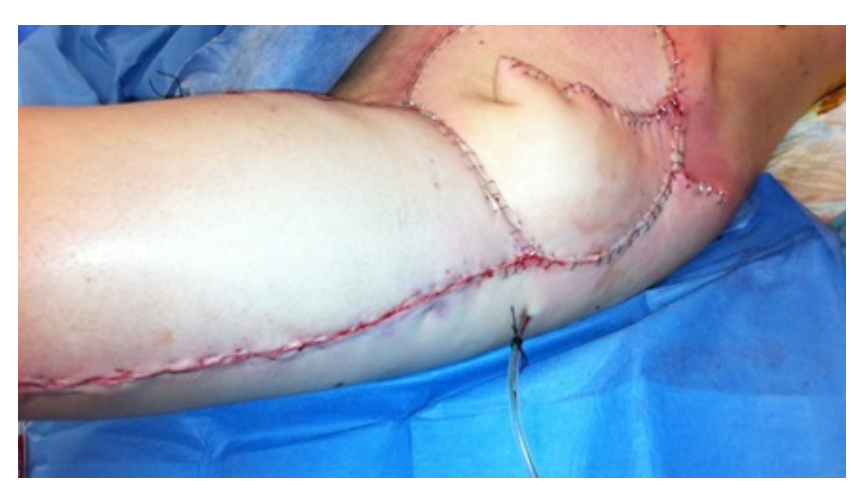

Figure 4 The lesion was excised and reconstructed with a Tensor Fascia Lata flap.

3 months later the patient developed recurrence in the anterior abdominal wall (Figure 5).

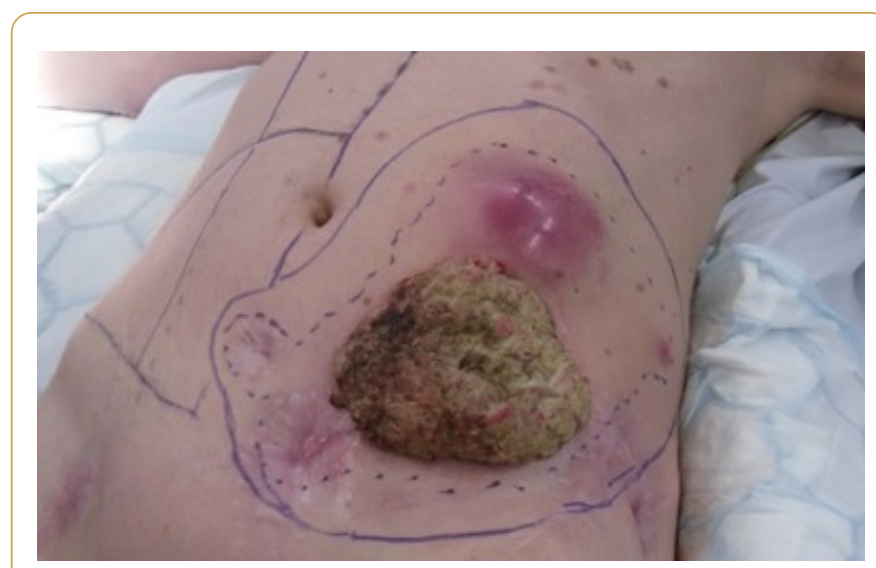

Figure 5 Patient developed recurrence in the anterior abdominal wall.

A multidisciplinary team meeting decided to proceed with further surgery. The patient underwent a wide local excision with a split skin graft reconstruction. One year after his initial procedure, the patient developed liver and lung metastases and further subcutaneous metastases in the left groin. He continued to have several more palliative resections for symptomatic relief before eventually succumbing to his disease. In our case report the patient presented with metastatic melanoma with no known primary lesion, although the primary melanoma may have spontaneously regressed. This melanoma recurred multiple times and progressed despite multiple surgical interventions and anti BRAF therapy. 